University of Nebraska - Lincoln

DigitalCommons@University of Nebraska - Lincoln

$1-15-2004$

\title{
The melting lines of model silicon calculated from coexisting solid-liquid phases
}

\author{
S. Yoo \\ University of Nebraska-Lincoln \\ Xiao Cheng Zeng \\ University of Nebraska-Lincoln, xzeng1@unl.edu \\ James R. Morris \\ Ames Laboratory (USDOE), lowa State University
}

Follow this and additional works at: https://digitalcommons.unl.edu/chemzeng

Part of the Chemistry Commons

Yoo, S.; Zeng, Xiao Cheng; and Morris, James R., "The melting lines of model silicon calculated from coexisting solid-liquid phases" (2004). Xiao Cheng Zeng Publications. 29.

https://digitalcommons.unl.edu/chemzeng/29

This Article is brought to you for free and open access by the Published Research - Department of Chemistry at DigitalCommons@University of Nebraska - Lincoln. It has been accepted for inclusion in Xiao Cheng Zeng Publications by an authorized administrator of DigitalCommons@University of Nebraska - Lincoln. 


\title{
LETTERS TO THE EDITOR
}

The Letters to the Editor section is divided into three categories entitled Notes, Comments, and Errata. Letters to the Editor are limited to one and three-fourths journal pages as described in the Announcement in the 1 January 2004 issue.

\section{NOTES}

\section{The melting lines of model silicon calculated from coexisting solid-liquid phases}

\author{
S. Yoo and X. C. Zeng \\ Department of Chemistry, University of Nebraska-Lincoln, Lincoln, Nebraska 68588 \\ James R. Morris \\ Metal and Ceramic Sciences Program, Ames Laboratory (USDOE), Iowa State University, Ames, \\ Iowa 50011
}

(Received 3 July 2003; accepted 22 October 2003)

[DOI: 10.1063/1.1633754]

The melting line is an important thermodynamic property of materials and can be calculated via computer simulation, given the intermolecular potential for the materials. Conventionally, the free-energy method has been mainly employed to calculate the melting line. ${ }^{1}$ Alternatives to the freeenergy method for calculating the melting line include simulation of the solid-liquid coexistence $e^{2,3}$ and nonequilibrium molecular dynamics (MD) method. ${ }^{4}$ Recently, one of us (Morris) and Song reported a detailed simulation procedure to calculate the melting line of the Lennard-Jones system. ${ }^{5}$ Specifically, the MD simulation consists of a preparation run and a production run. In the preparation run, the liquid and solid subsystems are separately equilibrated in a constant volume, particle number, and energy (NVE) ensemble. In the production run, the two subsystems are joined together and then the total system evolves towards the thermodynamic equilibrium in the $N V E$ ensemble. In this short note, we describe an improved preparation procedure for calculating melting lines of materials. This improved procedure is to address the anisotropic stress problem commented in the previous paper, ${ }^{5}$ that is, the principal components of stress tensor can be quite different during the production MD run if the initial lattice constant of the solid phase or the periodic boundary conditions are not carefully chosen. This anisotropy in stress can be quite substantial for hard solids such as the silicon, which renders the determination of the melting pressure difficult. We have tested the improved method with two empirical models of silicon: the Stillinger-Weber (SW) model $^{6}$ and the Tersoff- 89 model, ${ }^{7}$ as for both models the melting point at zero pressure has been determined previously via other methods. ${ }^{8-9}$

The improved simulation procedures are schematically illustrated in Fig. 1. In the preparation run, first, the simulation of a pure solid with the presumed stable lattice structure is taken in the isobaric-isothermal (NPT) ensemble by using the Nose-Andersen method ${ }^{10}$ at a temperature $T$ (assumed to be lower than the melting temperature $T_{m}$ ). The NoseAndersen method has the advantage of having a conserved quantity (the Nose-Andersen Hamiltonian) and therefore the coexisting system cannot evolve into a pure phase, even though both the liquid and solid phases are in equilibrium at the melting. After the solid system is fully equilibrated, the liquid phase is then generated by melting the solid phase at a higher temperature $T_{1}(\gg T)$ followed by a cooling to the original temperature $T$ at which the liquid is in the metastable state. Note that for the liquid simulation the $N P_{z} A T$ ensemble is used, where $P_{z}$ is the external pressure on the system along the $z$ axis (see Fig. 1) and $A$ is the area of the simulation cell normal to the $z$ axis. Because the internal pressure of the liquid phase is nearly independent of the shape of the simulation cell, the internal local stresses, $P_{x x}$, $P_{y y}$, and $P_{z z}$ for the liquid should be nearly the same. Next, in the production run, the two subsystems are brought into contact by joining the solid and liquid in the $z$-axis direction. The entire system is pre-equilibrated with a short run in the $N_{t} P_{z} A T$ ensemble (where $N_{t}=2 N$ ) to release large contact stress that might occur at the solid-liquid interface after the merge. Then, the coexisting solid and liquid are fully equilibrated in the constant-volume and constant-energy $N_{t} V E$ ensemble. The above simulation procedures can be repeated if the selected initial temperature $T$ is far below the $T_{m}$.

To test this improved simulation procedure for hard materials, we employed the SW and Tersoff- 89 models of silicon to evaluate their melting line. The potential function of the SW and Tersoff-89 model silicon can be found elsewhere. ${ }^{6,7}$ Note that for the SW system the energy is in units of $\epsilon(\epsilon=50 \mathrm{kcal} / \mathrm{mol}=2.16826 \mathrm{eV})$, the length is in units of $\sigma=2.0951 \AA$ and each MD time step is $0.15 \mathrm{fs}$, whereas for the Tersoff- 89 system the energy and length are in units of $\epsilon=1 \mathrm{eV}$ and $\sigma=1 \AA$ and each MD time step is 0.11 fs. For the production run, both the Si (111) and (100) surface were selected to be interfacing with the liquid phase. 


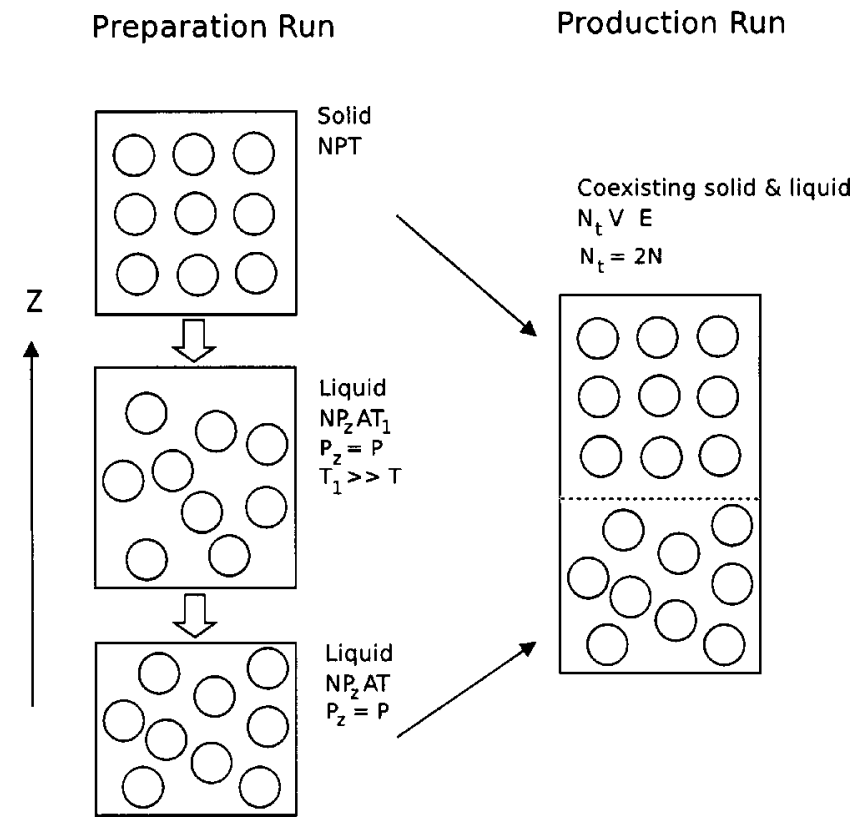

FIG. 1. A schematic illustration of the improved procedures to simulate solid-liquid coexistence.

For the former interfacial system the simulation cell contains 4800 atoms and has the size about $34 \AA \times 39 \AA \times 75 \AA$; for the latter the cell contains 5488 atoms and has the size about $38 \AA \times 38 \AA \times 76 \AA$. For the SW system 1000000 MD time steps were used for equilibration and another 1000000 steps were used for collecting data, while for the Tersoff- 89 system 3000000 steps for equilibration and another 1000000 steps for collecting data. In Figs. 2(a) and 2(b), we show a snapshot of the SW solid (111)/liquid and (100)/liquid interface, respectively. The former is very sharp and flat while the latter is much rougher due to the occurrence of the (111) faceting. ${ }^{3}$ In both cases, the location of the interface is nearly unchanged because the initial temperature selected is quite close to $T_{m}$. In Fig. 2(c), the three principal components of the stress vs MD time step are plotted for the coexisting SW

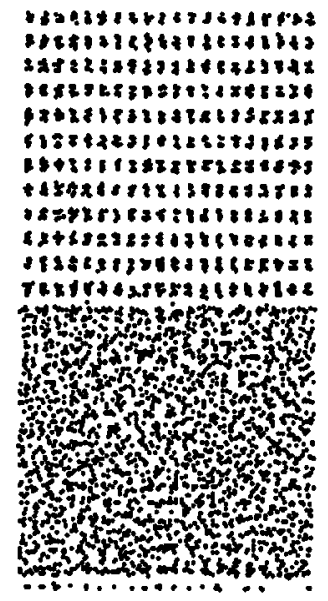

(a)

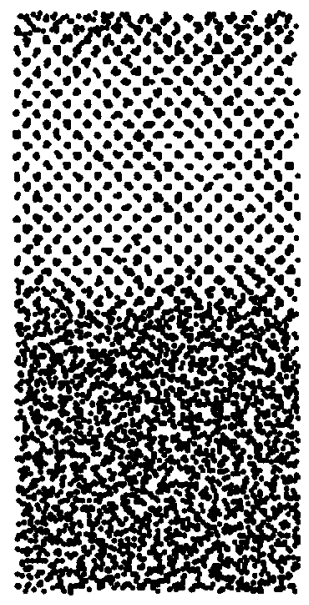

(b)

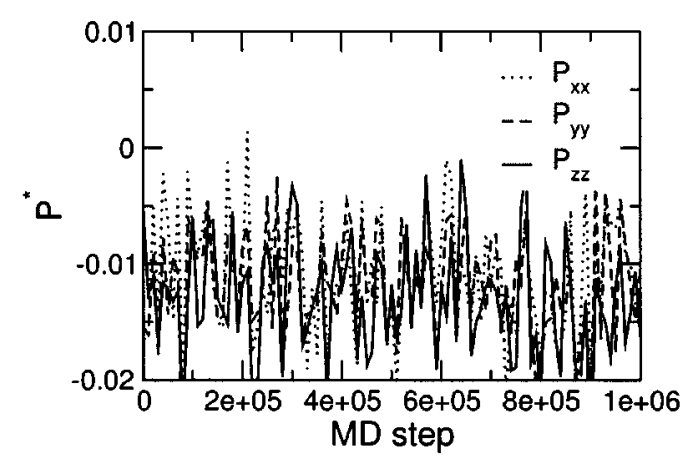

(c)

FIG. 2. A snapshot of equilibrated SW (a) solid (111)/liquid (b) solid (100)/ liquid interfacial system. (c) The three principal components of stress vs MD time step for the SW solid (100)/liquid system.

(100)/liquid system. Indeed, the three components are mostly the same during the data collection run.

The calculated $T_{m}$ at various pressures for both $\mathrm{SW}$ and Tersoff- 89 systems is given in Table I, where the calculated

TABLE I. The calculated melting temperatures and pressures. The data in the parentheses gives the root-mean-square error. The melting temperature at zero pressure is evaluated by interpolating the simulation results. Values of the melting pressure $P_{m}^{*}$ and temperature $T_{m}^{*}$ are in the reduced units of each silicon model.

\begin{tabular}{llll}
\hline \multicolumn{1}{c}{ Model } & \multicolumn{1}{c}{$P_{m}^{*}$} & \multicolumn{1}{c}{$T_{m}^{*}$} & $T_{m}(\mathrm{~K})$ \\
\hline SW(100) & $0.0201(36)$ & $0.0652(6)$ & $1641(15)$ \\
SW(100) & $-0.0052(14)$ & $0.0671(3)$ & $1688(8)$ \\
SW(100) & $-0.0118(13)$ & $0.0675(3)$ & $1698(8)$ \\
SW(100) & $-0.0288(9)$ & $0.0688(2)$ & $1731(5)$ \\
SW(100) (interpolation) & 0.0 & 0.0667 & 1678 \\
SW(111) & $0.0124(8)$ & $0.0664(1)$ & $1671(3)$ \\
SW(111) & $-0.0096(9)$ & $0.0678(1)$ & $1701(3)$ \\
SW(111) (interpolation) & 0.0 & 0.0672 & 1691 \\
SW (Ref. 8) & 0.0 & $0.0672(8)$ & $1691(20)$ \\
SW (Ref. 4) & 0.0 & $0.0671(11)$ & $1688(26)$ \\
Tersoff(100) & $0.0034(3)$ & $0.2187(14)$ & $2538(16)$ \\
Tersoff(100) & $-0.0084(2)$ & $0.2216(7)$ & $2572(8)$ \\
Tersoff(100) (interpolation) & 0.0 & 0.2212 & 2567 \\
Tersoff(100) (Ref. 9) & 0.0 & $0.2195(19)$ & $2547(22)$ \\
Tersoff(11) & $0.0025(2)$ & $0.2225(9)$ & $2582(10)$ \\
Tersoff(111) & $-0.0039(3)$ & $0.2231(5)$ & $2589(6)$ \\
Tersoff(11) (interpolation) & 0.0 & 0.2227 & 2584 \\
\hline \hline
\end{tabular}


$T_{m}$ at zero pressure is an interpolated result. For the SW model, the $T_{m}$ at zero pressure is almost the same as that calculated by Broughton and Li using a free-energy method. ${ }^{8}$ Note that the measured $T_{m}=1683 \mathrm{~K}$ at the ambient pressure. ${ }^{8}$ For the Tersoff- 89 model, the $T_{m}$ at zero pressure is very close to that determined by Cook and Clancy. ${ }^{9}$ However, the difference is larger than their reported error bar. Table I also shows that the $T_{m}$ at zero pressure predicted based on the solid (111)/liquid interface is slightly higher than that based on the solid (100)/liquid interface. However, the relative difference is less than $1 \%$. This difference may be due to the larger error bar entailed in the $T_{m}$ calculation based on the solid (100)/liquid interface because of the faceting and the resulting larger thermal fluctuation. It may be also due to some residual stress anisotropy in both interfacial systems. ${ }^{11}$ Finally, Table I shows that the predicted $T_{m}$ is lower at higher pressures. This result indicates that the slope of the melting line is negative ${ }^{12}$ for both SW and Tersoff-89 models, a result consistent with the measured one that the molar volume of silicon melt is smaller than that of the silicon solid upon melting. Only few materials in nature such as silicon and ice exhibit such unusual phenomena.

We thank Professor Xueyu Song for valuable discussions. This research was sponsored in part by the National Science Foundation, by the Division of Materials Sciences and Engineering, Office of Basic Energy Sciences, U.S. De- partment of Energy, under Contract No. W-7405-ENG-82 with Iowa State University, and by the Research Computing Facility at the University of Nebraska-Lincoln.

${ }^{1}$ D. Frenkel and B. Smit, Understanding Molecular Simulation: From Algorithms to Application (Academic, San Diego, 1996).

${ }^{2}$ J. R. Morris, C. Z. Wang, K. M. Ho, and C. T. Chan, Phys. Rev. B 49, 3109 (1994)

${ }^{3}$ U. Landman, W. D. Luedtke, R. N. Barnett et al., Phys. Rev. Lett. 56, 155 (1986).

${ }^{4}$ D. K. Chokappa, S. J. Cook, and P. Clancy, Phys. Rev. B 39, 10075 (1989).

${ }^{5}$ J. R. Morris and X. Song, J. Chem. Phys. 116, 9352 (2002).

${ }^{6}$ F. H. Stillinger and T. A. Weber, Phys. Rev. B 31, 5262 (1985).

${ }^{7}$ J. Tersoff, Phys. Rev. B 39, 5566 (1989); 38, 9902 (1988).

${ }^{8}$ J. Q. Broughton and X. P. Li, Phys. Rev. B 35, 9120 (1987).

${ }^{9}$ S. J. Cook and P. Clancy, Phys. Rev. B 47, 7686 (1993).

${ }^{10}$ D. C. Rapaport, The Art of Molecular Dynamics Simulations (Cambridge University Press, Cambridge, 1997).

${ }^{11}$ We also performed an MD simulation for the coexisting SW/(100)/liquid system without using the new procedure. In this particular test, the stress anisotropy amounts to $30 \%$. The calculated melting temperature at zero pressure is about $25 \mathrm{~K}$ below $1678 \mathrm{~K}$ (see Table I).

${ }^{12}$ Based on the SW (100) data (see Table I), we calculated $(d P / d T)_{m}=$ $-2.0 \pm 0.4 \times 10^{7} \mathrm{~J} / \mathrm{Km}^{3}$. According to the Clausius-Clapeyron equation, $(d P / d T)_{m}=L / T_{m} \Delta V$ where $L$ is the latent heat and $\Delta V$ is the volume change at the melting. Using data of $T_{m}, L$, and $\Delta V$ at $P=3.09$ $\times 10^{-6}$ bar (see Table $\mathrm{V}$ of Ref. 8), we obtained $L / T_{m} \Delta V=-2.08$ $\times 10^{7} \mathrm{~J} / \mathrm{Km}^{3}$ which is in good agreement with the $(d P / d T)_{m}$ value within the error bar. 